\title{
Bell's Palsy: A Need for Paradigm Shift?
}

\author{
Shraddha Jain ${ }^{1}$ Sunil Kumar² \\ ${ }^{1}$ Department of Otorhinolaryngology and Head and Neck Surgery, \\ Jawaharlal Nehru Medical College (JNMC), Datta Meghe Institute of \\ Medical Sciences (Deemed University, DMIMSU), Sawangi, Wardha, \\ Maharashtra, India \\ 2Department of Medicine, Jawaharlal Nehru Medical College (JNMC), \\ Datta Meghe Institute of Medical Sciences (Deemed University, DMIMSU), \\ Sawangi, Wardha, Maharashtra, India
}

\begin{abstract}
Address for correspondence Shraddha Jain, MBBS, MS, PhD (ENT), Department of Otorhinolaryngology and Head and Neck Surgery, Jawaharlal Nehru Medical College, DMIMSU, Sawangi, Wardha 442004, Maharashtra, India (e-mail: sjain_med@yahoo.co.in).
\end{abstract}

Ann Otol Neurotol ISO 2018;1:34-39

\author{
Abstract \\ Keywords \\ - Bell's palsy \\ - peripheral neuropathy \\ - idiopathic facial \\ paralysis \\ - herpes simplex virus \\ - autoimmunity \\ - genetic
}

Introduction Bell's palsy (BP) is considered to be idiopathic facial palsy. Some schools of thought believe it to be a part of polyneuropathy, whereas other scientists have other plausible explanations for the condition and all these were reviewed.

Methods The literature on BP was retrieved using the Cochrane Database of Systematic Reviews, PubMed, and Google Scholar. Keywords and phrases used during the search included "Bell's palsy," "polyneuropathy," "anatomical factors," and "autoimmunity."

Results There are only few studies that have tried to find out the association of BP with peripheral nerve conduction abnormalities. In most studies conducted, positive correlation was found. There are many theories of its etiology, with the reactivation of herpes simplex virus isoform 1 and/or herpes zoster virus (HZV) from the geniculate ganglia being the most strongly suspected cause.

Conclusion In the future, large studies will be required to determine the association between peripheral neuropathy and BP and elucidate the real etiology in every case. There will also be a need to determine whether BP should still be considered idiopathic and isolated.

\section{Introduction}

Bell's palsy (BP) is universally accepted as isolated, sudden, peripheral facial paralysis of unknown etiology. Whether it should actually be considered isolated and idiopathic needs to be determined. Most BP cases could represent an overt sign of a subclinical polyneuropathy. There are reports that idiopathic facial paralysis is part of a generalized subclinical polyneuropathy or mononeuritis multiplex. ${ }^{1-3}$ Some consider BP to be a part of cranial polyneuropathy. ${ }^{4-5}$ Various etiologies have been implicated for BP, which is otherwise considered as idiopathic. Different causes that have been suggested include viral agents, autoimmunity, as a part of acute subclinical diabetic polyneuropathy and vascular factors, among others. ${ }^{6-10}$

\section{Historical Aspects}

Bell's palsy is named after the British physician Sir Charles Bell, who described the onset, physical findings and course of the disease in 1821. However, some historical records have shown that Nicolaus A. Friedrich, an 18th-century professor of medicine of Wurzburg, may have been the first to report idiopathic peripheral facial nerve paralysis, 23 years before Bell's report. ${ }^{11}$ He gave an account of three middle-aged adults with a similar onset of acute or subacute unilateral facial paralysis, which improved with time, in his De paralysis Musculorum Faciei Rheumatica, first published in 1798 in the German medical literature. ${ }^{12}$

\section{Clinical Features}

Although BP is the most common cause of facial palsy (80\%), with an incidence of 20 to 30 cases per 100,000 individuals, it is still considered a diagnosis of exclusion..$^{13}$ In other words, only after exclusion of all known causes of infranuclear facial palsy is it labeled as BP and has maintained its historical description given by Sir Charles Bell. The facial paralysis in BP typically is abrupt in onset or gradually worsens over 2 to 3 days. The slow 
progression of paralysis over weeks to months rules out BP and is consistent with a primary facial nerve tumor or a malignant process. The presence of other symptoms, such as facial twitching, hearing loss, vestibular dysfunction, otorrhea, and severe, unrelenting otalgia, disfavors the diagnosis of BP as some underlying attributable pathology can be found in these cases. ${ }^{13}$

\section{Anatomy of the Facial Nerve (Cranial Nerve VII)}

Knowledge of the anatomical course and intricacies of the facial nerve, that is, seventh cranial nerve (CN VII) will help us better understand the pathophysiology of BP. The facial nerve is motor to the muscles of face, the stapedius, stylohyoid, and posterior belly of digastric muscles. The parasympathetic fibers from the facial nerve are carried by the greater superficial petrosal nerve to supply the lacrimal gland, and by the chorda tympani nerve to the submandibular and sublingual salivary glands. Hence involvement of facial nerve makes all these fibers susceptible to paralysis.

The CN VII enters the temporal bone at the internal auditory canal (IAC), then it passes through the fallopian canal, and finally exits through the stylomastoid foramen and gives extratemporal branches ( - Fig. 1). The intratemporal course of the facial nerve is divided into four segments, namely meatal, labyrinthine, tympanic, and mastoid. It enters the IAC at its medial end at the porus and emerges at the lateral end (fundus) after merging with its sensory division, the nervus intermedius. The IAC portion of the nerve (meatal segment) is approximately 8 to $10 \mathrm{~mm}$ long. The labyrinthine segment (4 $\mathrm{mm}$ in length)-the first segment of the bony fallopian canal-is the narrowest and shortest portion of the canal. The meatal foramen, the entry point of facial nerve into the labyrinthine segment of its fallopian canal, is the narrowest portion of the entire canal measuring approximately $0.68 \mathrm{~mm}$ in diameter. ${ }^{14} \mathrm{~A}$ dense arachnoid band also encircles the nerve at the lateral end of the IAC contributing to the anatomic "bottleneck" that further constricts the nerve when it is edematous, in conditions such as BP. ${ }^{14}$ Thus, the meatal foramen and labyrinthine segment of the nerve play a pivotal role in the pathophysiology of facial paralysis, as is discussed later in this review. The fallopian canal provides a

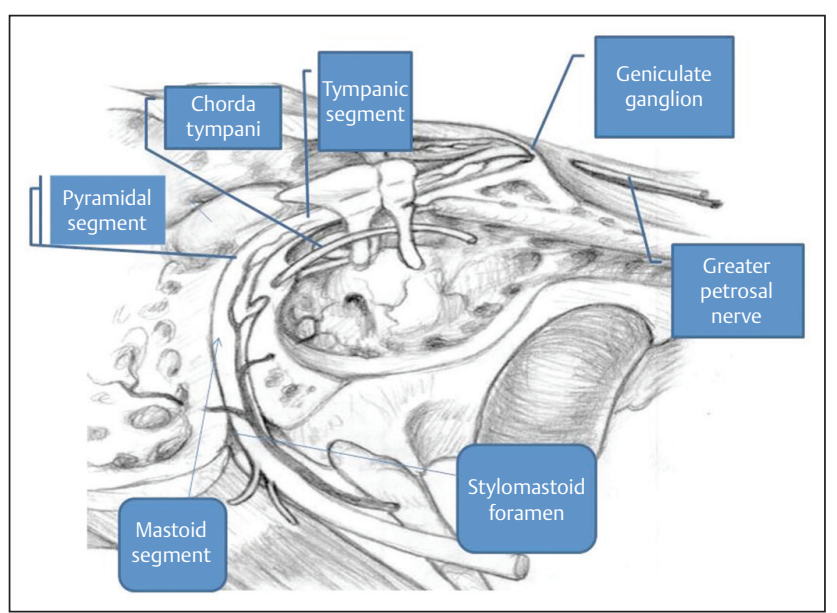

Fig. 1 Intratemporal anatomy of facial nerve. bony covering for the facial nerve that is longer than that of any other nerve and acts as a "double-edged sword." On the one hand, it provides protection to the nerve but also makes it vulnerable to palsy, due to entrapment neuropathy. The fallopian canal has congenital gaps, or dehiscences, which again render the facial nerve liable to injury.

\section{Mechanism and Etiology}

Though it is considered idiopathic, the search for mechanism of BP has been the subject of research for decades, with the underlying cause still remaining unclear despite several proposed theories. The authors have discussed different proposed mechanisms and etiologies for BP in the following sequence: viral, environmental factors, autoimmunity, as part of polyneuropathy, vascular factors, ischemic (compressive) factors, pregnancy, and anatomic factors.

From the aforementioned text, it is evident that the probable mechanism for BP could be the compression of facial nerve along its narrow segment at the lateral end of the IAC, secondary to any type of inflammatory edema. ${ }^{12}$ Initially, inflammation causes only a temporary loss of sensory or motor function, but it may result in permanent nerve degeneration later due to compression in the fallopian canal. ${ }^{12}$

\section{Viral Etiology}

The most accepted theory is that BP is a peripheral neuropathy due to viral infection that causes swelling of the cells of the facial nerve. BP has been thought to result from reactivation of latent herpes virus infection in the geniculate ganglia and its subsequent migration to the facial nerve. ${ }^{12,15}$ Majority of the current literature supports the concept of herpes simplex virus (HSV)-mediated inflammation leading to nerve compression and subsequent facial paralysis. ${ }^{6,12,15} \mathrm{HSV}$ reactivation within the geniculate ganglion leading to inflammation and compression of the nerve at the meatal foramen has been the most accepted pathogenetic mechanism of BP.

The evidence obtained from laboratory studies of animals and humans have linked HSV-1 to facial nerve paralysis, one of them being isolation of HSV-1 DNA from the endoneural fluid of the facial nerve by polymerase chain reaction (PCR) during the acute phase of BP, as done by Murakami et al. ${ }^{16}$ Others have detected HSV-1 in the saliva of $29 \%$ of the BP patients by PCR. ${ }^{17}$ When degree of facial palsy was studied, it was observed that negative PCR for HSV-1 cases had a lower grade of facial palsy than positive PCR cases, indicating that virus-positive cases had greater neural involvement. However, this observation was not supported by facial nerve electroneurography findings. ${ }^{17}$ Apart from HSV-1, herpes zoster virus (HZV) has also been implicated as the likely causative agent, with this form of infection being more severe, as the virus spreads across the nerve by means of satellite cells. ${ }^{12}$

Inactivated intranasal influenza (flu) vaccine has also been linked with the causation of BP by Mutsch et al, who reported a strong temporal and specific association between the risk of developing BP in patients who received the vaccine as compared with the control groups who did not receive the vaccine. ${ }^{18}$ 


\section{Doubts over Viral Etiology}

Recently, doubts have been raised over the causal association of HSV/HZV with BP. A recent study explored the presence of viral genomic DNA of HSV-1 and 2, human herpes virus (HHV)-6A/B, as well as varicella zoster virus (VZV) by performing PCR in facial muscle biopsy specimens, Scarpa's ganglion, geniculate ganglion, and tear fluid, from patients with BP and in controls without the disease. ${ }^{19} \mathrm{HSV}-1, \mathrm{HSV}-2$, or VZV genomic DNA were not detected in Scarpa's ganglion or muscle biopsy results in control as well as BP patients. On analysis of tear fluid samples from control patients and patients with acute BP, HHV-6A could be detected in tear fluid samples of both control (40\%) and BP (30\%) patients. The viral DNA was detected even in the controls, from their geniculate ganglion preparations, the incidence of HSV-1 and VZV genomic DNA being 86 and $43 \%$, respectively. ${ }^{19}$

Hence, instead of mere presence of HSV genomic DNA along the facial nerve in patients with acute BP, the identification of an active replicating virus would be a better investigation and has not yet been performed.

\section{Environmental Factors Acting as Possible Triggers of the Disease}

Low humidity, dry indoor air, cold temperatures, ultraviolet radiation, and coinfections of the upper respiratory tract have been implicated as possible triggers of this disease by causing reactivation of latent HSV virus. ${ }^{20}$ However, another study has found higher incidence of BP in summer and autumn. ${ }^{21}$ This could be related to ultraviolet exposure leading to virus activation. Thus environmental factors are controversial, though most favor cold temperatures as the most important environmental trigger for $\mathrm{BP}^{22}$ Another study from Peru observed workplace clustering of cases of BP, but extensive epidemiologic survey and serologic analysis were unable to find out any common identifiable risk factor among all cases. ${ }^{23}$ They concluded that there is limited additional benefit in conducting an environmental exposure assessment for identifying a cause in outbreaks of BP and that future investigations should prioritize focus on search for common infectious etiology.

\section{Autoimmunity}

Some authors feel that BP may be an autoimmune demyelinating cranial neuritis or a mononeuritic variant of Guillain-Barré syndrome (GBS) with cell-mediated immunity against nerve myelin antigens. In BP and GBS, a viral infection or the reactivation of a latent virus is believed to provoke an autoimmune reaction against peripheral nerve myelin components, leading to the demyelination of the nerves. ${ }^{5,7}$ It has been suggested that they both represent an inflammatory demyelinating neuritis in which $\mathrm{BP}$ can be considered a mononeuritic variant of GBS. 7,12

Aviel and colleagues found a significant decrease in the peripheral blood T-lymphocyte percentage and an increase in B-lymphocyte percentage in BP patients during the acute phase of the disease. ${ }^{24}$ However, there was no change in the levels of immunoglobulins (IgG, IgM), complement (C3, C4), or antiviral antibodies to herpes simplex and zoster, Epstein-Barr virus (EBV), cytomegalovirus, adenovirus, influenza, and mumps. The immunologic data of BP showed similar changes in peripheral blood lymphocyte subpopulations as described in the course of several demyelinating diseases, such as in acute exacerbations of multiple sclerosis and during the acute stage of GBS, suggesting that BP may be an autoimmune demyelinating cranial polyneuritis that may be caused by a preceding viral infection. ${ }^{7,24}$

\section{As Part of Polyneuropathy}

Apart from etiology, the other point of controversy is that it is not always "isolated" and may be a part of more generalized subclinical polyneuropathy of different origin. . $^{1-3,7,8}$

The viral agents may trigger cranial or generalized polyneuropathy in BP.2 Therefore, BP could be a part of subclinical viral polyneuropathy. The electromyographic studies obtained from the leg muscles in patients with BP have shown changes similar to those occurring in peripheral nerve lesions, and the changes correlated well with the degree of facial palsy. ${ }^{8}$ Abnormal values have also been reported in the maximal motor nerve conduction velocities and motor terminal latencies of the median and peroneal nerves in other study. ${ }^{3}$ BP as a part of acute cranial or peripheral polyneuritis could be caused probably by reactivation of the HSV, and the dysfunction of the motor cranial nerves (V, VII, and X) may represent inflammation and demyelinization rather than ischemic compression. ${ }^{5}$ The mechanism for postviral subclinical polyneuropathy in BP could be autoimmunity leading to demyelinating polyneuropathy similar to GBS. ${ }^{7}$

It could also represent manifest mononeuropathy in acute subclinical diabetic polyneuropathy. ${ }^{25}$ It has been found that the part of facial nerve distal to the chorda tympani is susceptible to microangiopathy of diabetes, which causes localized facial nerve ischemia (infarction?) and edema, further aggravated due to the rigid fallopian canal at that area. The facial nerve, in this respect, is similar to the other nerves commonly affected by diabetic mononeuropathy; all traverse an enclosed space that makes them especially vulnerable to the effects of ischemia. Thus subclinical involvement of other cranial and peripheral nerves in BP is not necessarily of an infective etiology, but it can also occur due to diabetes mellitus, even of recent onset. ${ }^{25}$ In diabetic patients with BP, focal neuropathies are more frequent, whereas sensory neuropathies with small nerve fiber involvement are less frequent. ${ }^{26}$

\section{Vascular Factors}

Diabetes, hypertension, and hypercholesterolemia, all have been associated with microangiopathy leading to microcirculatory failure of the vasa nervosum and are potential causative and poor prognostic factors in BP. There are different studies, some supporting the association whereas others refuting it. In some, no significant correlation was found between the severity of palsy and comorbid disease, including hypertension, diabetes, and hyperlipidemia. ${ }^{21}$ 
The finding of significantly lower taste impairment in diabetics than in nondiabetics with BP supports the hypothesis of a vascular rather than a metabolic etiology. ${ }^{10}$ This supports the theory that vessels supplying the distal portion of the facial nerve are probably more affected in the diabetic patients as taste sensation is preserved in most. ${ }^{25}$

In diabetic patients, there is chronic nerve ischemia due to reduction in endoneurial oxygen, compromised nerve blood flow, and epineurial arteriovenous shunting. Preexisting nerve ischemia and injury, as well as abnormalities of endoneurial and epineurial vessels, could be a reason for higher grade of facial palsy in diabetic patients presenting with $\mathrm{BP}{ }^{27}$ In a study by Riga et al, hemoglobin $\mathrm{A} 1 \mathrm{c}\left(\mathrm{HbA}_{1 \mathrm{c}}\right)$ values were found to be significantly correlated with the severity of BP, whereas hypertension and hypercholesterolemia were not found to be correlated with the severity of BP. ${ }^{27}$ Efficient glycemic control is important for the prevention as well as the treatment of peripheral neuropathies in diabetic patients. Therefore, BP of viral etiology presenting in a diabetic patient will tend to have a worse grade on presentation and could have a more proximal origin.

Thus, impairment of taste sensation in a diabetic could point toward viral etiology favoring antiviral treatment, whereas preservation of taste is indicative of diabetic mononeuropathy.

The patients with abnormal $\mathrm{HbA}_{1 \mathrm{c}}$ values seem to completely recover at the same frequency as patients with normal $\mathrm{HbA}_{1 \mathrm{c}}$ values.

\section{Ischemic (Compressive) Factors}

One of the theories for BP is "compressive theory."28 The best possible explanation for most of the cases labeled as BP appears to be "entrapment neuropathy" that results from inflammation, edema, and strangulation of the facial nerve within the osseous fallopian canal, the most common site being the meatal foramen. ${ }^{29}$ The observation that the onset and development of facial palsy occur during sleep and is noticed mostly in the morning points toward the ischemia as the cause due to reduction in circulatory dynamics at night. ${ }^{30}$ This could be a secondary ischemia due to postinflammatory edema of the nerve.

The site of nerve edema has been studied by various authors. ${ }^{29,31}$ In cases of HSV reactivation, inflammation occurs in the geniculate ganglion of the facial nerve and progresses distally or proximally. ${ }^{31}$ The meatal foramen, being the narrowest portion of the facial canal, subjects the nerve to maximal pressure, even if the degree of inflammation change is the same. ${ }^{29}$ In a study comparing operative findings with magnetic resonance imaging (MRI) in patients with BP, in all the patients studied, the edema proceeded from the geniculate ganglion toward the peripheral area as time progressed. ${ }^{31}$ Intraoperatively, the edema of the labyrinthine segment was mainly observed in the acute phase; the tympanic segment edema was seen in the subacute phase, 3 to 9 weeks after symptom onset. ${ }^{31}$ However, as time progressed, the edema remained only in the labyrinthine and geniculate ganglion and after the ninth week, no swelling of the nerve was observed in about one-third of the patients. ${ }^{31}$ In another study, the edema of labyrinthine segment of the facial nerve was seen in all patients with enhancement of the labyrinthine segment on MRI, whereas less correlation was found between the MRI findings and actual nerve edema (only 43\%) in tympanic segment. ${ }^{29}$ The explanation given for this is that the presence of greater amount of normal arteriovenous plexus in the tympanic segment than the labyrinthine or geniculate ganglion, so that even without the presence of nerve edema, enhancement can be observed.

Among all cranial nerves, facial nerve is the only nerve that travels in a bony canal. Nerve dysfunction may result more easily as the facial nerve swells within the confines of the noncompliant bony facial canal. Endoneural pressure then increases, and neural vasculature is compressed, leading to ischemia, impairment of axonoplasmic flow, and axonal degeneration. ${ }^{32}$ This conclusion is confirmed on findings in perioperative and MRI studies, as well as the significantly better outcome after corticosteroid administration. . $2,29,31^{2}$

\section{Pregnancy}

The physiologic changes that occur in late pregnancy or the immediate puerperium have been thought to predispose to BP. ${ }^{33}$ However, some feel that altered susceptibility to HSV reactivation during pregnancy is the most likely explanation for concentration of cases in the third trimester. ${ }^{34}$

\section{Anatomical Factors}

Viral infection is relatively common, but BP is not so common. Hence, there has been a constant search for various anatomic factors in bony facial canal that may predispose to the development of paralysis.

Vianna et al found no significant difference in the diameter of the facial nerve or canal between the affected and unaffected sides in patients with unilateral BP, suggesting that there is no anatomical predisposition favoring the development of compressive edema on one side versus the other. ${ }^{32}$ They observed that the mean diameter of the bony facial canal in the tympanic and mastoid segments on both sides (BP side and contralateral side) of the BP group was significantly smaller than the control group, and there was no significant difference between them in the labyrinthine segment. The limitation of the study was use of postmortem specimens.

This was in contrast to another study by Kefalidis et al, in which the bony measurements at both sites were significantly smaller in the affected side. ${ }^{35}$ They suggested that an asymmetry between the right and left fallopian canal might be a necessary pathogenetic mechanism for the development of a facial nerve edema into BP in the narrower fallopian canal. By high-resolution computed tomography (CT) with multiplanar reconstruction technique, Murai et al also found that both in the labyrinthine and horizontal segments, the mean cross-sectional area of the facial nerve canal was significantly 
smaller on the affected side of patients with BP than on the unaffected side. ${ }^{36}$ No significant difference was found between the affected and unaffected sides in the cross-sectional area of the facial nerve canal in the mastoid segment. The labyrinthine segment, being the narrowest segment in the facial nerve canal, makes the facial nerve vulnerable, especially in the labyrinthine segment of the facial nerve canal, and a narrow facial nerve canal may be one of the risk factors for BP.

In another study, the authors wanted to find why majority of the paresis are suprastapedial, that is, why the entrapment of the nerve mainly occurs in the proximal part of the canal. For this, anatomical measurements of the facial canal diameter and cross-sectional area made in 12 temporal bones by use of a computer program proved that the width of the canal is smaller at its proximal part. ${ }^{37}$ The nerve is thicker as it contains more nerve fibers, causing discrepancy between the nerve diameter and the surrounding bony walls in the suprastapedial part of the of the canal, which, in cases of a swollen nerve after inflammation, results in facial palsy. ${ }^{37} \mathrm{CT}$ of the temporal bone should be undertaken in every case of BP as it enables not only measurement of exact length and thickness of individual segments of the facial nerve canal but also to view variable anatomic variations in the intratemporal facial canal. Anatomical variations, such as dehiscent facial canal, may predispose the nerve to inflammatory processes or neural compression injury. ${ }^{38-40}$

\section{Treatment}

From the above review, it is evident that postinflammatory edema of the nerve that runs in a narrow bony canal is the best explanation for BP, and hence corticosteroids are now universally recognized to be beneficial in improving the outcome of BP. However, it is yet to be resolved whether or not a combination of antiviral agents and corticosteroids result in a better rate of complete facial recovery compared with corticosteroids alone. Some clinical trials have shown the efficacy of antiviral agents combined with corticosteroids in increasing the rate of complete recovery in BP, whereas others have found no added benefit of antivirals to corticosteroids alone in producing complete facial recovery. ${ }^{12}$ Because of the possible viral or autoimmune pathogenesis of BP, a combination therapy of antivirals and oral corticosteroids should be considered, if patients present early. ${ }^{41,42}$ This has been supported by placebo-controlled trials that demonstrated full recovery in a higher percentage of patients treated with an antiviral drug in combination with prednisolone than with prednisolone alone. .3.44 $^{2}$

The authors observed, from their unpublished data, that when systemic features point toward viral illness and associated with a severe degree of facial palsy, antivirals should be added to hasten the recovery. Considering its course in the bony canal, the authors propose injectable steroids, such as methylprednisolone, in doses similar to those for treatment of acute spinal cord injury, $20 \mathrm{mg}$ or more of methylprednisolone/kg of body weight in a bolus dose over a 15-minute period. ${ }^{45}$ Immediate excellent recovery has been observed in these patients from grades 5 to 1 . Antivirals should be added as high-dose steroids could flare up the viral infection. More studies are needed to confirm this.

\section{Conclusion}

This review article has attempted to emphasize the fact that "Bell's palsy" as an entity does not have any significance in today's era. Lack of sufficient investigations makes us label it idiopathic and isolated, as many times we do not investigate for the presence of polyneuropathy. Facial nerve is the only nerve in the bony canal and hence manifests itself, whereas others might go unnoticed. Moreover, underlying anatomic factors may also be contributory, which need sophisticated imaging.

The key points can be summarized as follows:

- BP in most cases is not idiopathic or isolated; rather, it may be a part of viral, diabetic, or demyelinating neuropathy, being manifest due to the typical anatomical course and the variations in temporal bone anatomy.

- Instead of proposing various theories for BP, the authors should enlist different etiologies for it.

- In every case, if exact cause is identified, treatment may be better.

- Search for subclinical peripheral neuropathy should be done in every case, and it may help identify the viral or diabetic etiology and treatment decision for antiviral treatment.

- Radiological assessment in the form of high-resolution $\mathrm{CT} / \mathrm{MRI}$ of the temporal bone should form part of assessment protocol in every case.

\section{Areas of Further Research}

- The search for identification of an active replicating virus in BP rather than isolation by PCR would be a better investigation.

- The precise peripheral nerve antigens, against which cell-mediated immunity acts, have not been identified as yet.

- Monoclonal antibodies and/or T-cell immunotherapy might provide more specific form of treatment in the management of BP.

- Research on imaging protocols for identifying facial nerve edema and anatomical variations.

\section{References}

1 Abdel-Baki F, Moghazi H, Eassa A, Talaat F. Peripheral nerve involvement in acute Bell's palsy. J Laryngol Otol 1988;102(5):447-448

2 Tunç T, Tarhan E, Oğuz H, Şafak MA, Kutlu G, İnan LE. Is Bell's palsy a component of polyneuropathy? Mediterr J Otol 2006;2(1):1-6

3 Bueri JA, Cohen LG, Panizza ME, Sanz OP, Sica RE. Peripheral nerve involvement in Bell's palsy. Arq Neuropsiquiatr 1984;42(4):341-345

4 Benatar M, Edlow J. The spectrum of cranial neuropathy in patients with Bell's palsy. Arch Intern Med 2004;164(21):2383-2385 
5 Adour KK, Byl FM, Hilsinger RL Jr, Kahn ZM, Sheldon MI. The true nature of Bell's palsy: analysis of 1,000 consecutive patients. Laryngoscope 1978;88(5):787-801

6 Kennedy PG. Herpes simplex virus type 1 and Bell's palsy-a current assessment of the controversy. J Neurovirol 2010;16(1):1-5

7 Greco A, Gallo A, Fusconi M, Marinelli C, Macri GF, de Vincentiis M. Bell's palsy and autoimmunity. Autoimmun Rev 2012;12(2):323-328

8 Sandstedt P, Hydén D, Odkvist L. Bell's palsy-part of a polyneuropathy? Acta Neurol Scand 1981;64(1):66-73

9 Yanagihara N, Hyodo M. Association of diabetes mellitus and hypertension with Bell's palsy and Ramsay Hunt syndrome. Ann Otol Rhinol Laryngol Suppl 1988;137:5-7

10 Paolino E, Granieri E, Tola MR, Panarelli MA, Carreras M. Predisposing factors in Bell's palsy: a case-control study. J Neurol 1985;232(6):363-365

11 Bird TD,, Nicolaus , A . Friedreich's description of peripheral facial nerve paralysis in 1798. J Neurol Neurosurg Psychiatry 1979;42(1):56-58

12 Zandian A, Osiro S, Hudson R, et al. The neurologist's dilemma: a comprehensive clinical review of Bell's palsy, with emphasis on current management trends. Med Sci Monit 2014;20:83-90

13 Gantz BJ. Idiopathic facial paralysis. In: Current Therapy in Otolaryngology-Head and Neck Surgery. BC Decker; 1987:62-66

14 Fisch U, Esslen E. Total intratemporal exposure of the facial nerve. Pathologic findings in Bell's palsy. Arch Otolaryngol 1972;95(4):335-341

15 Schirm J, Mulkens PS. Bell's palsy and herpes simplex virus. APMIS 1997;105(11):815-823

16 Murakami S, Mizobuchi M, Nakashiro Y, Doi T, Hato N, Yanagihara N. Bell palsy and herpes simplex virus: identification of viral DNA in endoneurial fluid and muscle. Ann Intern Med 1996;124(1 Pt 1):27-30

17 Lazarini PR, Vianna MF, Alcantara MP, Scalia RA, Caiaffa Filho $\mathrm{HH}$. Herpes simplex virus in the saliva of peripheral Bell's palsy patients. Rev Bras Otorrinolaringol (Engl Ed) 2006;72(1):7-11

18 Mutsch M, Zhou W, Rhodes P, et al. Use of the inactivated intranasal influenza vaccine and the risk of Bell's palsy in Switzerland. N Engl J Med 2004;350(9):896-903

19 Linder T, Bossart W, Bodmer D. Bell's palsy and herpes simplex virus: fact or mystery? Otol Neurotol 2005;26(1):109-113

20 Campbell KE, Brundage JF. Effects of climate, latitude, and season on the incidence of Bell's palsy in the US Armed Forces, October 1997 to September 1999. Am J Epidemiol 2002;156(1):32-39

21 Zohrevandi B, Monsef Kasmaee V, Asadi P, Tajik H. Report of 121 cases of Bell's palsy referred to the emergency department. Emerg (Tehran) 2014;2(2):66-70

22 de DJ, Prim MP, Madero R, Marcos S, Gavilan J; de DJ. Effect of atmospheric factors on the incidence of Bell's palsy. Eur Arch Otorhinolaryngol 2002;259(1):53-55

23 Reaves EJ, Ramos M, Bausch DG. Workplace cluster of Bell's palsy in Lima, Peru. BMC Res Notes 2014;7:289

24 Aviel A, Ostfeld E, Burstein R, Marshak G, Bentwich Z. Peripheral blood T and B lymphocyte subpopulations in Bell's palsy. Ann Otol Rhinol Laryngol 1983;92(2 Pt 1):187-191

25 Pecket P, Schattner A. Concurrent Bell's palsy and diabetes mellitus: a diabetic mononeuropathy? J Neurol Neurosurg Psychiatry 1982;45(7):652-655
26 Kiziltan ME, Akalin MA, Sahin R, Uluduz D. Peripheral neuropathy in patients with diabetes mellitus presenting as Bell's palsy. Neurosci Lett 2007;427(3):138-141

27 Riga M, Kefalidis G, Danielides V. The role of diabetes mellitus in the clinical presentation and prognosis of Bell palsy. J Am Board Fam Med 2012;25(6):819-826

28 de Diego JI, Prim MP, Gavilán J. Aetiopathogenesis of Bell's idiopathic peripheral facial palsy [in Spanish] Rev Neurol 2001;32(11):1055-1059

29 Kim IS, Shin SH, Kim J, Lee WS, Lee HK. Correlation between MRI and operative findings in Bell's palsy and Ramsay Hunt syndrome. Yonsei Med J 2007;48(6):963-968

30 Kanoh N, Nomura J, Satomi F. Nocturnal onset and development of Bell's palsy. Laryngoscope 2005;115(1):99-100

31 Yanagihara N, Honda N, Hato N, Murakami S. Edematous swelling of the facial nerve in Bell's palsy. Acta Otolaryngol 2000;120(5):667-671

32 Vianna M, Adams M, Schachern P, Lazarini PR, Paparella MM, Cureoglu S. Differences in the diameter of facial nerve and facial canal in bell's palsy-a 3-dimensional temporal bone study. Otol Neurotol 2014;35(3):514-518

33 McGregor JA, Guberman A, Amer J, Goodlin R. Idiopathic facial nerve paralysis (Bell's palsy) in late pregnancy and the early puerperium. Obstet Gynecol 1987;69(3 Pt 2):435-438

34 Vrabec JT, Isaacson B, Van Hook JW. Bell's palsy and pregnancy. Otolaryngol Head Neck Surg 2007;137(6):858-861

35 Kefalidis G, Riga M, Argyropoulou P, et al. Is the width of the labyrinthine portion of the fallopian tube implicated in the pathophysiology of Bell's palsy?: a prospective clinical study using computed tomography. Laryngoscope 2010;120(6):1203-1207

36 Murai A, Kariya S, Tamura K, et al. The facial nerve canal in patients with Bell's palsy: an investigation by high-resolution computed tomography with multiplanar reconstruction. Eur Arch Otorhinolaryngol 2013;270(7):2035-2038

37 Dawidowsky K, Branica S, Batelja L, Dawidowsky B, KovaćBilić L, Simunić-Veselić A. Anatomical study of the facial nerve canal in comparison to the site of the lesion in Bell's palsy. Coll Antropol 2011;35(1):61-65

38 Kim J, Jung GH, Park SY, Ko SH, Lee WS. Anatomical consideration of the temporal bone as a pathogenesis of Bell's palsy. Med Hypotheses 2011;77(5):705-707

39 Baxter A. Dehiscence of the fallopian canal. An anatomical study. J Laryngol Otol 1971;85(6):587-594

40 Nager GT, Proctor B. The facial canal: normal anatomy varieties and anomalies: II. Trans Am Otol Soc 1983;70:61-77

41 Lee HY, Byun JY, Park MS, Yeo SG. Steroid-antiviral treatment improves the recovery rate in patients with severe Bell's palsy. Am J Med 2013;126(4):336-341

42 Billue JS. Bell's palsy: an update on idiopathic facial paralysis. Nurse Pract 1997;22(8):88, 97-100, 102-105, quiz 106-107

43 Hato N, Yamada H, Kohno H, et al. Valacyclovir and prednisolone treatment for Bell's palsy: a multicenter, randomized, placebo-controlled study. Otol Neurotol 2007;28(3):408-413

44 Numthavaj P, Thakkinstian A, Dejthevaporn C, Attia J. Corticosteroid and antiviral therapy for Bell's palsy: a network meta-analysis. BMC Neurol 2011;11:1

45 Bracken MB. Steroids for acute spinal cord injury. Cochrane Database Syst Rev 2012;1:CD001046 